\title{
LEGION BASED WEED EXTRACTION FROM UAV IMAGERY
}

\author{
Ahmed Mateen ${ }^{1,2, *}$ and Qingsheng Zhu ${ }^{1}$ \\ ${ }^{1}$ College of Computer Science, Chongqing University, China; ${ }^{2}$ Computer Science Department,University of \\ Agriculture Faisalabad, Pakistan. \\ *Corresponding author's e-mail: ahmedmatin@hotmail.com
}

\begin{abstract}
Multispectral images are the types of images which consist of many different bands of data for further processing. Vegetation Indexing has Different bands which are combined in the multispectral images and are used to accentuate the vegetated areas. In this one combination is most commonly used LIKE Ratio Vegetation Indexing (RVI). But using UAV imagery this problem has been solved because in UAV the area covered or captured by the cameras would be very wide so that the whole plot or crop can be viewed or captured from the UAV imagery. Legion is a Model used to do Scene Analysis Task, Image Segmentation, Object Selection and Speech Segregation. In this Research work we used the Legion Network approach to Detect or Segregate the Unwanted Crops or Weed to increase the crop productivity by Detecting or Segregating the Unwanted Crops or Weed. We used UAVDJI Phantom 4 having RGB Sensor and Tetracam NIR Sensor to collect the data set. Then we used ImageJ and MATLAB for applying Legion Model algorithm to process and analyze the multidimensional images using linear differential equations which solves the correlation problem of synchronization and Segregation of UAV based crop images to Detect the Unwanted crops.
\end{abstract}

Keywords: Legion Network, Image Segmentation, Ratio Vegetation Indexing, Multidimensional Images.

\section{INTRODUCTION}

Every country's progress is heavily reliant on agricultural growth or productivity. Agriculture now plays a vital role in the economies of every country on the planet. As a result, the precision agriculture field has been introduced (Buayuai et al., 2017). This is a new field in which the use and benefits of information technology in the field of farming and crop growth are being introduced for the first time. Many different techniques and algorithmic approaches are used in Precision Agriculture to increase crop productivity in any country. The main purpose of using IT in farming is also to increase crop productivity (Mandal et al.,2014). As a result, many algorithms and designs are used, such as early weed detection (unwanted crop detection in any crop), crop modelling, vegetation indexing, pest detection, and many others. These are all Smart farming approaches to increasing crop yield in any country (Cantelli et al., 2013).In the future, the demand for food will increase day by day, so in order to manage the entire world's food supply, new ways and methods to increase crop yield, such as smart farming, will be developed (Weis et al., 2009) Many benefits can be obtained by using smart farming, such as time management, reduced water and chemical use, and early pest and weed detection, allowing us to increase crop yield productivity (Li et al., 2011; Quiles et al., 2011). Now, UAV-based Crop Detection and Weed Detection is a critical step in this process. UAV (Unmanned Aerial Vehicle) is a type of drone that flies over crops at various heights and takes images of crops from various angles and heights (Grichuk et al., 2011). These images are processed in preparation for crop detection and separation or crop modelling in the future (Sa et al.,2018; Brik et al., 2011). In the field, there are some very difficult places or environments where UGV is working to assist the human operator because there is a very difficult task or work for human operator to operate on the very large fields, so for this reason, Unmanned Ground Vehicle has been used for SMART FARMING in the previous few decades (Tang et al., 2016; Wang et al., 1999). However, despite the many benefits of the UGV System, this is not a good strategy in today's world. Recently, UAV imagery approaches or methods have become very popular in this field, not only because of their high resolution and temporal resolution, but also because of their ability to obtain or take spectra and special data at the same time (Rao et al., 2016; Ursino et al., 2003). As a result, in recent years, Unmanned Aerial Vehicle (UAV) technology has been used in place of the UGV (Girau et al., 2007). One significant disadvantage of the UGV system is that only a limited view of crops can be obtained or covered by the cameras, creating a difficult situation (Pérez-Ortiz et al., 2016). In this regard, this article provides a comprehensive review of unmanned aerial imagery applications for natural resource management (Aravind et al., 2015). We concentrate on image-based processes and describe the current state of unmanned aerial imagery applications, including challenges and future opportunities (Pea et al., 2013; Ishida et al., 2018; Wang et al., 2015).

1) The end user application developed to manage agricultural practices. 
2) Timely implementations of different agricultural processes are being proposed.

As previously stated, the LEGION Model used in this study to detect weed unwanted crops is entirely based on the oscillatory correlation theory (Paikekari et al., 2016). There are many types of solvers which are used in the MATLAB to solve the differential equations (Saleem et al., 2015). Ode51, ode 45 are the most important two functions which are used (Burgos-Artizzu et al., 2011; Samseemoung et al., 2012). Now the theory is that make one global inhibitor which is used in this model for the purpose of desynchronizations (Vibhute et al., 2012). In next step make the local inhibitors which is based on the local features of the objects and established the connections between different local inhibitors Sankaran et al., 2015). Every local inhibitor is connected with three other local inhibitors to make the network (Hung et al., 2014). For this reason, this model is also called oscillatory correlation network model (Chen et al., 2014; Erena et al., 2016). As we know the LEGION Model is the mathematical model which are based on the Linear single order Differential equations in MATLAB these equations are solved by the functions which are called solver functions we used ODE45 functions in general to solve any kind of differential equations (Pena et al., 2015). ODE means Ordinary Differential equations (Matese et al., 2015). The main questions is that for which purpose Ordinary Differential equations are used in LEGION model (Louargant et al., 2017). There are two types of images used in this process one is gray and other is colored (Peña et al., 2015). In Gray images there is no luminance effect and no color information. In the other hand the information or characteristics of plants and weeds are required and processed the image of any crop accurately then convert the colored images into gray images (Hernandez et al., 2015). When image is converted into gray scale the image is in the the binary form so the segmentations of weed and background is very easy (Zhang et al., 2012). After converting image into gray scale many types of noise like dummy white pixels, plants shadows which can affect the accuracy of processing of images (Zongjian et al., 2008). So, for this reason noise reduction or removal of the noise is very important for the further processing of the images. So, for noise reduction different techniques can be used in this scenario used the Gaussian noise reduction (Peña et al., 2013).we focus on a related but slightly different phenomenon: the emergence of commercial drones. These drones are designed, built and used by individuals, businesses, and organizations. Though commercial drones owe much of their development to their military counterparts, most designs do not resemble the larger and more expensive surveillance drones (Cantelli et al., 2013). The purpose of segmenting the image into plantand background pixels is to detect the amount of plant material within a specific area. If the amount of plant material reaches a specific threshold, that area is targeted for herbicidal spay application (Ishida et al., 2018). The percentage of greenness, weeds and the normalized difference vegetation index (NDVI) wereobtained at different altitudes and compared for their relative performance. In the low-dynamic mode of image data acquisition (tractor driven crane- attachment), the tractor speed was maintained constant and low to avoid excessive jerks during image acquisition. In the high-dynamic mode of image data acquisition (helicopter- mounted platform), a commercially available unmanned helicopter was selected. The helicopter weighed approximately $6 \mathrm{~kg}$ with a payload capacity of $5 \mathrm{~kg}$ and was flown by a skilled Person with a radio transmitter functioning at a frequency of $42 \mathrm{MHz}$ and with a range of 1-2 km radius (Mandal et al., 2014). The spatial distribution of weeds has often been ignored in weed management because the techniques for measuring the spatial variation of weeds have so far not been implemented. Nevertheless, there is a general agreement that herbicide use can be reduced significantly when applied site specifically. The majority of farmers still need to spray herbicides uniformly across the field, due to the lack of technology for automatic weed sampling techniques and site-specific application technology (Louargant et al., 2017). A major step towards a practical solution for site- specific weed management is the development of precise and powerful sampling techniques to automatically and continuously determine in-field variation of crop cover and weed seedling populations (Hernandez et al., 2015).

\section{MATERIALS AND METHODS}

We use the LEGION model in this study to separate and recognise areas of weeds and crops from the field photos. Legion is essentially the mathematical model, which means that the entire operation of this type is based on oscillatory correlation network models or phases. The full name of this model is a global oscillatory Network Model locally exciting. The Oscillational Network models, as we know, are therefore the smallest unit in this model. As we know, it is important or hard to group similar characteristics and characteristics in the segmentation process. Legion is a simple math model based on the two simple linear equations of first order. Thus, these equations apply to the whole data set or images in the Legion model in order to achieve superior outcomes. This model is used in the main to detect various objects in phases synchronisation and desynchronization. We know that every object that can be detected has its own characteristics, since each object has its own distinct characteristics. The same steps, albeit in the form of oscillations, are therefore followed in this model.

Legion Model Equations: As we know the Legion Model is a mathematical model basedon the first order linear differential equations which solves thecorrelation problem. So there two main equations which are involved in the process of synchronization and desynchronization. These two equations are the first order differential equations. And We also know 
these equations arethe main equations of the LEGION Model the whole theory is based on these two differential equations. This model is based upon mathematical relationship. LEGION Model is based on single oscillator for i simple form of oscillator and

loop between the excitatory unit xi and inhibitory unit yi. As it can be represented with following Eq. (1) and Eq. (2) first order differential equations.

$$
\begin{aligned}
& \frac{d x_{i}}{d t}=3 x_{i}-x_{i}^{2}+2-y_{i}+\rho+I_{i}+S_{i} \\
& \frac{d y_{i}}{d t}=\epsilon\left[\gamma\left(1+\tanh \left(\frac{x_{i}}{\beta}\right)\right)-y_{i}\right]
\end{aligned}
$$

As in these two equations different parameters are used and know we explain these parameters or effect of these parameters on the model. $I i$ represents the external stimulus and $\rho$ represents the amplitude of the guassian noise term. If the amplitude of noise is greater the results are affected. Si represents the coupling term of the different oscillators in the network. The noise is included in every system and also guassian noise is added to measure the robustness of the system. The most significant attribute of any DRONE technology is the flight time of the drone, which is a distinguishing attribute of every drone. The flight time of each kind of drone varies, and drone technology is that which increases flight time. When the drone takes off, the flight time is recorded. As a result, the requirements for flight time vary from domain to domain.

In the agricultural area, the drone's flight period should be at least 20 minutes in order to gather crop photographs. So phantom 4 pro is the model that provides all of the attributes required for the agricultural area, including flight time. The Flight time of phantom 4 pro model is very good and effective explained in table 1.
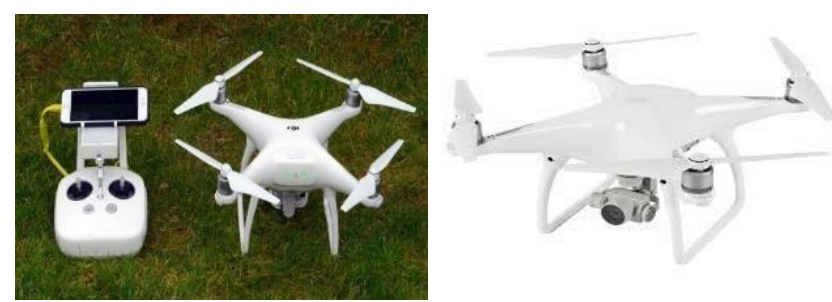

Figure 1. Phantom DJI 4 UAV (Pérez-Ortiz et al., 2016)

DJI phantom 4 pro is the most recent model; prior models such as phantom 3, phantom 2, and many others are also employed in this industry; however, the flight timing of each model varies (Saqib et al., 2018). Prior versions of the Phantom DJI Series, such as the Phantom 4 and Phantom 3, had relatively short flight times however the flight time of the Phantom 5 Quad copter DJI is relatively long. So, in comparison to other versions, this model has the longest flight time, and another advantage is that separate batteries are included with the gadget; these batteries are rechargeable as shown if Figure 1-2..

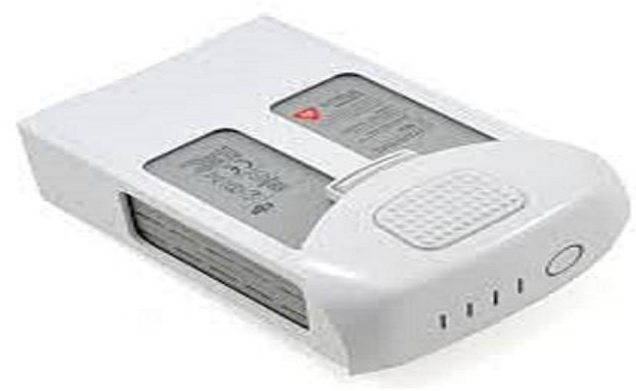

Figure 2. Rechargeable battery of DJI Phantom 4 UAV (Ishida et al., 2018)

Table 1. Specifications of DJI Phantom 4 Quadcopter.

Sr. Specifications

1. Max Flight Time

2. Max Speed

3. Altitude Range

4. Image Size

5. Supported SD Card

6. Battery Capacity

7. Voltage

8. Max. Weight

9. Obstacle Sensing System

10. Vedio Output Port

11. Max Ascent Speed

12. Max Descent Speed

28 Minutes
S- Mode $20 \mathrm{~m} / \mathrm{s}$
$0-33$ feet $(0-10 \mathrm{~m})$
$5000 * 4000$
Max Capacity $64 \mathrm{~GB}$
$5350 \mathrm{mAh}$
17.4 Volt
$1388 \mathrm{~g}$
Yes $($ Rang 0.7 to $25 \mathrm{~m})$
HDMI $($ Yes $)$ USB $($ Yes $)$
S-mode $5 \mathrm{~m} / \mathrm{s}$
S-mode $20 \mathrm{~m} / \mathrm{s}$

There are numerous tools and softwares with variable version specs that can be used (Saqib et al., 2018). Table 2 shows the tools that are used to alter data at various phases of the LEGION algorithm.

Table 2. Software's List Used in this Research.

\begin{tabular}{|c|c|c|c|}
\hline \multicolumn{3}{|c|}{ 1. Microsoft Excel } & $\begin{array}{l}\text { Data Saving in the form of } \\
\text { pixels }\end{array}$ \\
\hline \multirow[t]{3}{*}{2.} & MATLAB & R2017b & Image Processing Toolbox \\
\hline & R2017b & & Computer Vision Toolbox \\
\hline & & & Image Acquisition Toolbox \\
\hline \multirow[t]{2}{*}{3.} & ImageJ & $1.52 \mathrm{a}$ & Histogram \\
\hline & & & Color Spreads \\
\hline 4. & UAV & \multicolumn{2}{|c|}{ DJI Phantom 4} \\
\hline 5. & RGB Sensor & \multicolumn{2}{|c|}{ TCS34725 } \\
\hline 6. & Tetracam NIR & \multirow{2}{*}{\multicolumn{2}{|c|}{230218}} \\
\hline & Sensor & & \\
\hline
\end{tabular}
Sr. Tools/Software Version Component/Module

\section{ALGORITHMIC DESCRIPTION FOR WEED} DETECTION BASED ON FEATURE EXTRACTION

Below is our algorithm on feature extraction for image segmentation and phase synchronization for weed detection. Image as initial condition in ode 45

Read Image and show image 
i = imread('newtest.png');

imshow(i);

1. First Convert the image into binary

imshow $(\mathrm{g})$;

$$
\mathrm{g}=\mathrm{im} 2 \mathrm{bw}(\mathrm{i}) \text {; }
$$

2. for double precision

$\mathrm{g}=\mathrm{im} 2 \mathrm{double}(\mathrm{g})$;

3. image store in column vector

initVec $=[\mathrm{g}]$

initVec = initVec(:);

4. Image resizing

$\mathrm{i}=$ reshape (dataset, $[140,114])$

5. Initialize variables

$\mathrm{I}=0.1 ; \quad$ Rho $=0.1$;

Eta $=0.02$;

Gamma $=8$

Beta $=0.1$

Epsilon $=0.02$;

Phi $=3.0$;

6. Defining timespan for solving function

[t1 tf] So t1 which represents the initial time and

tf which represents the final time

$\mathrm{T} 1=0$;

$\mathrm{Tf}=100$

StepSize $=0.1$;

7. Equation Solving with parameters

$[\mathrm{T}, \mathrm{Y}]=$ ode45(@LegionODE,

(T1:StepSize:Tf), initVec);

figure, $\operatorname{plot}(\mathrm{Y})$;

$\operatorname{plot}(\mathrm{Y}(:, 1), \mathrm{Y}(:, 2))$;

8. phase synchronization and desynchronization objects

function $\mathrm{dy}=\operatorname{LegionODE}(\mathrm{t}, \mathrm{y})$

$\mathrm{m}=95$;

$\mathrm{n}=84$;

total $230000 * 2=4600 \mathrm{~m} * \mathrm{n}, 1)) *(\mathrm{y}(1: \mathrm{m} * \mathrm{n}, 1))$

.*(y(1:m*n,1)) +2 ;

9. Image segmentation and crop and weed identified

$[\mathrm{t}, \mathrm{x}]=$ ode45(@ fname, tspan, xinit, options)

- Fname is the function name used to evaluate the righthand function. This is the function where we will incorporate the first-order ode system..

- Tspan is the vector defining the beginning and end limits of integration and the size of our time steps. I.e. if we integrate $\mathrm{t}=0$ to $\mathrm{t}=10$ and wish to take 100 steps, then tspan $=[0: 0.1: 10]$ or tspan=linspace $(0,10,100))$.

- Initial conditions vector xinit. Make sure the order matches the order used to write $\mathrm{y}, \mathrm{z}$ and their $\mathrm{x}$-derivatives. Also note that if $\mathrm{x}$ consists of 5 variables, we require 5 initial conditions.

- $\quad \mathrm{t}$ is an independent variable with a solution array $\mathrm{x}$ computed. This vector doesn't always match tspan above since ode 45 does some play about with step sizes to maximise both accuracy and efficiency (taking smaller steps when the problem varies rapidly and bigger steps where it's reasonably consistent). However, t's length is the same as tspan's.

As previously discussed, the LEGION model is essentially the rate of change of anything because differential equations are used in places where the rate of change is necessary to determine(Saqib et al., 2018). In this section, we will first implement the LEGION algorithms on random vectors based on initial conditions rather than image data, and then we will test the LEGION algorithms in Fig.3-4 .

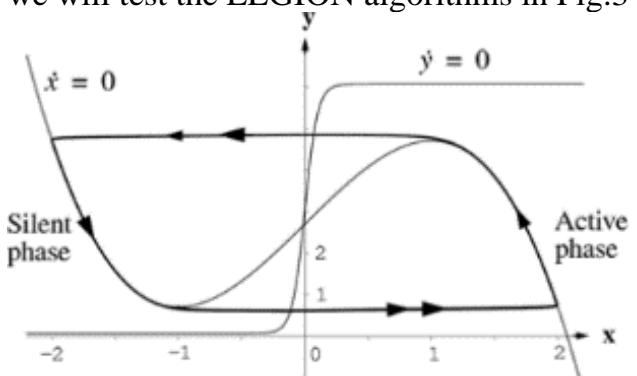

Figure 3. Simple Graph of Legion Model (Girau et al.,2007)

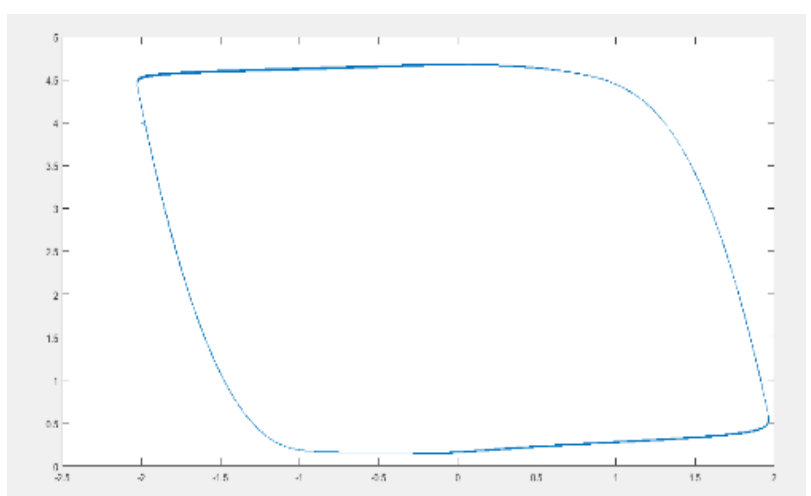

Figure 4. Implemented Legion graph on random initial condition

Apply Model on Green Extraction Original Crop Image Apply Model on Green Extraction Original Crop Image Now the next step of this research work is to apply this legion model on the real images which we captured to segregate or detect the weeds from the crop image. As we know the dimension of the original image of field which we used is $5000 * 4000$ and the total column vector of image is comprised of over the $20,000,000$ which is very large data and used for the purpose of detection. So, to solve the issue of memory, we cleared the memory of previous vector and again wrote the next column vector, which produced the image against this column vector. So, this is the strategy which was used to solve the issue of memory. 


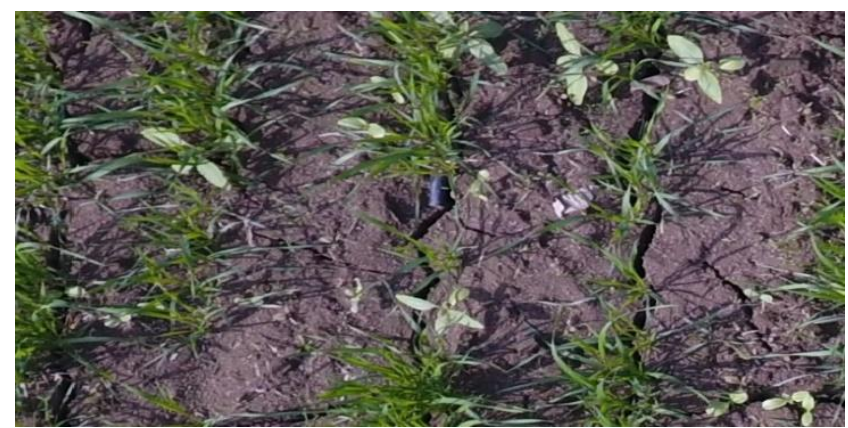

Figure 5. Original cropped image of weed

We cropped the image to decrease image size and then converted this into the column vector for applying legion model . Cropped image is shown in figure 5 . After converting

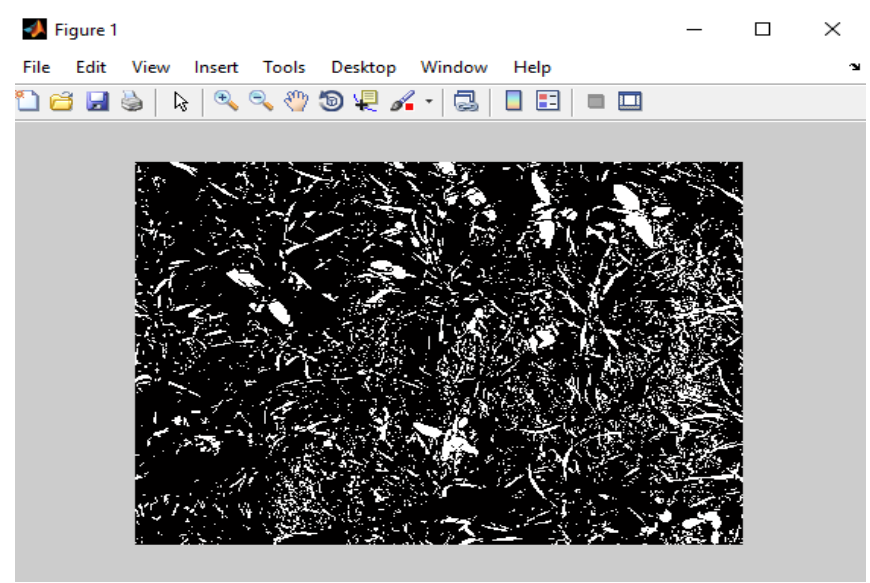

Figure 6. Image in binary form

values are in single column vector. We cropped the image to decrease image size and converted this into the column vector for applying legion model. we applied the

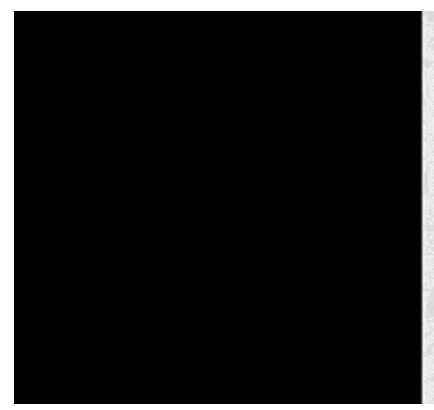

Figure 7. Detected weed at last time step

legion model on this image to detect the weed rows in the field. So, for this reason, same process which we applied on the simple image was also applied on this image but in this scenario the image size is very large. The memory management is very important in this scenario. The use of memory is discussed above. First of all, we used image as a data and converted image data into column vector and converted the image into binary form because legion model is applied on binary form. Image in binary form can be seen in figure 6-7.

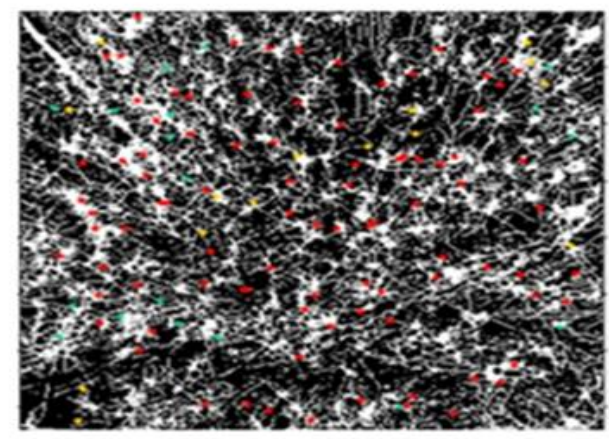

(a)

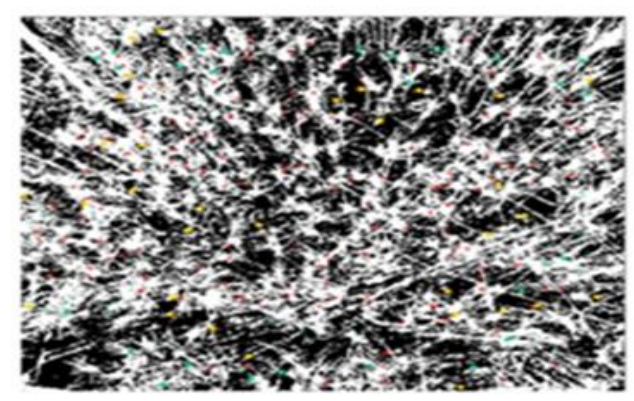

(b)

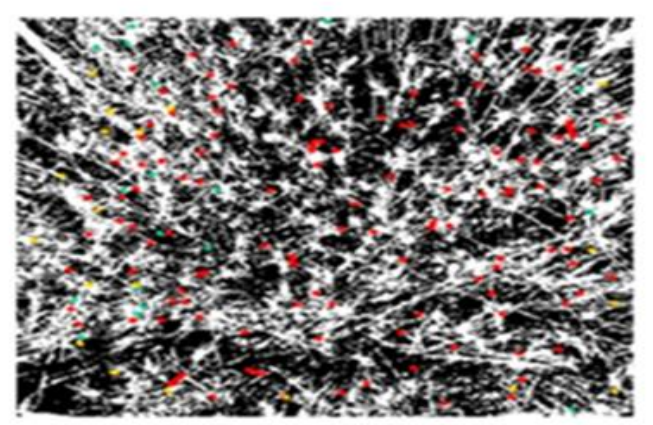

I(c)

Figure 8. Segmentation results (a) edge detection method; (b) region growth method; (c) proposed method

After converting we made the initial condition of this vector and after setting the required parameters, which are used in the legion model (which are constant like gamma, rho and many more now), we called the legion node function which basically solves the equations of the legion model to detect 
the weed and pass the initial condition of image vector as a parameter in the ode45 function.

\section{PERFORMANCE ANALYSIS AND COMPARISON}

We analyze the effect of image segmentation on the accuracy of recognition with different methods. we first use the proposed method herein to segment the images and determine the number of wheat and weed patches. intuitively. The number of wheat and weed patches obtained after the segmentation of different methods is calculated statistically as shown in table3.

We provide more appropriate indicators to confirm by obtaining $\mathrm{R}^{2}$ of proposed method, Edge detection method and region growth method. From figure 8-10.

Table 3. Inter-region contrast values and segmentation accuracy for different segmentation

\begin{tabular}{lcc}
\hline \multicolumn{1}{c}{ Method } & $\begin{array}{c}\text { Inter region } \\
\text { contrast }\end{array}$ & $\begin{array}{c}\text { Segmentation } \\
\text { Accuracy }\end{array}$ \\
\hline Proposed method & 0.487 & 0.947 \\
Edge detection method & 0.645 & 0.885 \\
Region growth method & 0.570 & 0.795 \\
\hline
\end{tabular}

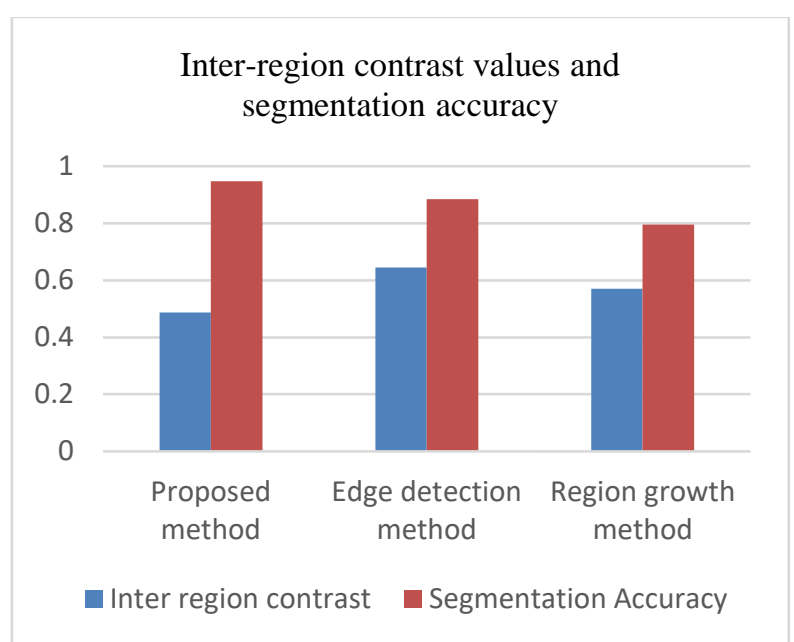

Figure 9. Inter-region contrast values and segmentation accuracy

We can see that after comparing with other segmentation methods, the $\mathrm{R}^{2}$ between the proposed segmentation results is closer to 1 which means this method could provide a statistic value much closer to the real value and has a higher accuracy and good robustness in different areas

After comparing numerical accuracy, the segmentation results are quantitatively analyzed by segmentation accuracy table 3 . The inter-region contrast index is a measurement of image segmentation quality based on inter-regional contrast where the smaller value represents the better segmentation effect.
From Table 3 , we can draw the conclusion that compared with the other segmentation methods, our proposed method could achieve more accurate image segmentation.
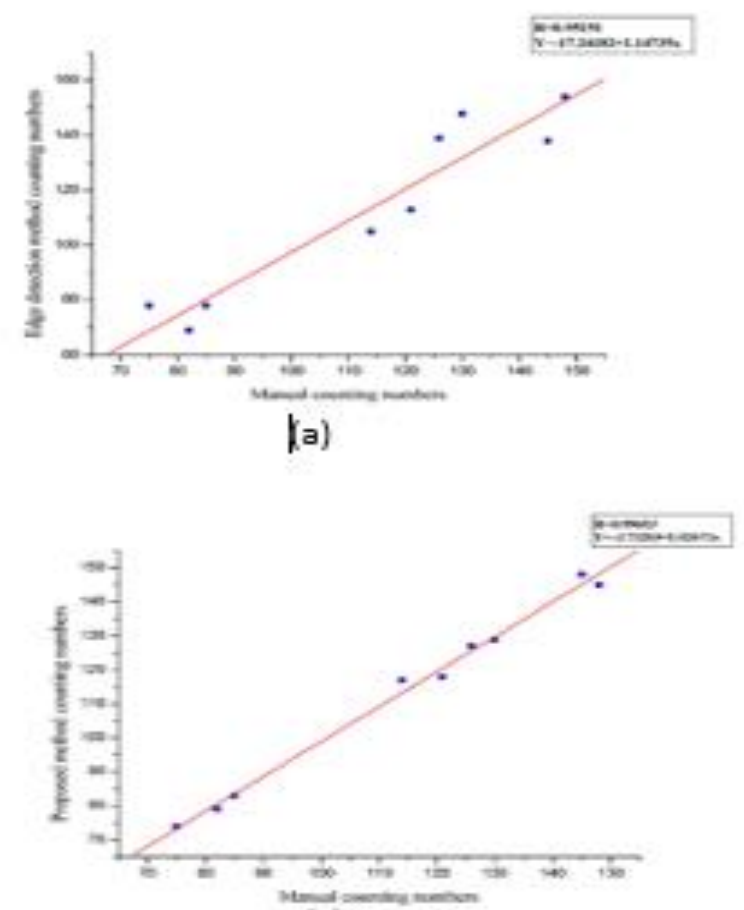

(b)

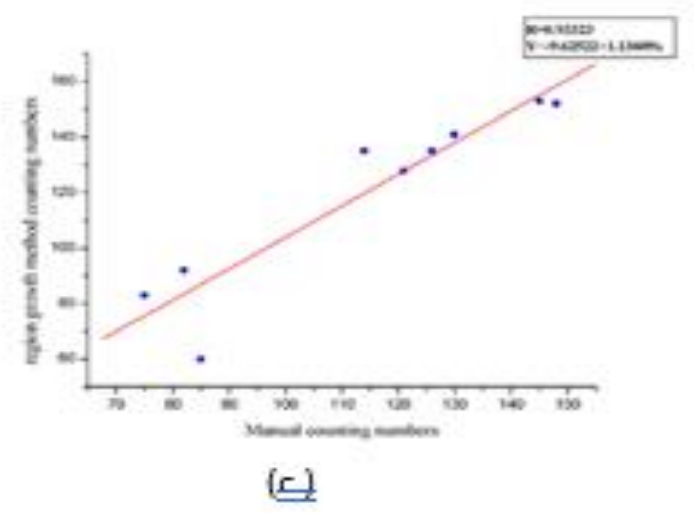

Figure 10. Image Segmentation results compared with manual results

Conclusion: Legion network approach to detect or segregate the unwanted crops or weed to increase the crop productivity. This model is basically used for scene analysis task, object selection, image segmentation and speech segregation. Here in the research, that model is used for segregation of UAV based real crop images to detect the unwanted crops. In this model, features of an object were grouped i-e same pixels grouped into visual object and after that these same features objects are segregated from other objects based on oscillatory correlation. 


\section{AI-based Agriculture}

In this technique, an object has a single phase which represents the oscillators and more than one objects have different phases and represent different phase oscillator group. Legion network is not similaor to other neural networks because the basic unit is neural oscillators which represent the one unit or object. Connectivity between different oscillators in this approach consists of excitation between locally bounded oscillators and comparing these locally connected oscillators with global inhibited features (globally inhibitor). After getting the results, it can achieve fast and robust synchronization between the locally connected oscillators group and desynchronize between different phases oscillators group.

We applied this algorithmic model in the actual image data set captured through UAV as our goal was to detect the weed from photographed farmland photos. To apply this on images data in the initial condition, we passed the image as a parameter but, firstly we converted the image into binary form and after that, converted the whole image into the column vector format. We applied this legion model on this image data having parameters values $I=2.0$ The differential equations were solved through the following parameters. $€=0.02, \alpha=5.0, \beta=0.1, K=50, \Theta x$ $=-0.5, \Theta z=0.1$ and $\phi=3.0$ after image segmentation. So, image size should be small because the large image data is taking more time to solve the problem. As we know the dimension of the original image of field (which we used) is $5000 * 4000$ and the total column vector of image is comprised over the $20,000,000$ which is very large data used for the purpose of detection so, So we cropped the image to reduce the size of image and now the dimension of image was $700 * 450$ and we know that converted this into the column vector for applying legion model after converting 760000 values are in single column vector. Now the dimension became $760000 * 1000$ when $\mathrm{t} 1=1$ and $\mathrm{tf}=100$ and step $=0.1$. In these images it is clearly seen that weeds ( unwanted crops ) are detected through many steps and the results are based on the 0.1 -time step and time range from 0 to 100 . In small period of time, oscillators having same patterns synchronize and oscillator of different groups desynchronize. Moreover , separated parts that are associated together would be identified as a single object. The simulations indicate that this approach has the capability to quickly segment an image having different patterns and shows the nature of robustness which are very likely to bind disconnected image regions associated with each other.

Acknowledgement: We acknowledge the valuable support of Dr. Muhammad Ahsan Latif and his students in completing this work.

\section{REFERENCES}

Buayaui, P., T. Kantanukul , C. Leung and K. Saikaew, K. 2017. Boundary Detection of Pigs in Pens based on Adaptive Thresholding using an Integral Image and Adaptive Partitioning. CMU J. Nat. Sci. 16:145-156.
Mandal, M., M. Ali, A. Amin, S. Masum and H. Mehraj. 2014. Assessment of different weed control methods on growth and yield of wheat. IJAAR. 5:65-73.

Cantelli, L., M. Mangiameli, C.D. Melita and G. Muscato. 2013. UAV/UGV cooperation for surveying operations in humanitarian demining. Safety, Security, and Rescue Robotics (SSRR), IEEE International symposium on (SSRR);pp.1-6Weis, M. and R. Gerhards. 2009. Detection of weeds using image processing and clustering. Borni. Agrartech. Beric. 69:138-144.

Li, C. and Y. Li. 2011. Fast and robust image segmentation by small-world neural oscillator networks. Cogn. Neurodynamics. 5:209-220.

Quiles, M.G., D. Wang, L. Zhao, R.A. Romero and D.S. Huang. 2011. Selecting salient objects in real scenes: An oscillatory correlation model. Neural Netw. 24:54-64.

Grichuk, E., M. Kuzmina and E. Manykin. 2011.Object selection in visual scene via oscillatory network with controllable coupling and self-organized performance. Opt. Memory \& Neural Netw. 20:113-119.

Sa, I., Z. Chen, M. Popović, R. Khanna, F. Liebisch, J. Nieto and R. Siegwart. 2018. weedNet: Dense Semantic Weed Classification Using Multispectral Images and MAV for Smart Farming. IEEE Robotics Autom. Lett. 3:588-595.

Birk, A., B. Wiggerich, H. Bülow, M. Pfingsthorn and S. Schwertfeger. 2011. Safety, security, and rescue missions with an Unmanned Aerial Vehicle (UAV).J Intell.

Robotic Syst. 64:57-76.

Tang, J.-L., X.-Q. Chen, R.-H. Miao, and D. Wang. 2016. Weed detection using image processing under different illumination for site-specific areas spraying. Compt. Electron. Agr. 122:103-111.

Rao, B., A.G. Gopi and R. Maione. 2016. The societal impact of commercial drones. Technol. Soc. 45:83-90.

Ursino, M., G.-E. La Cara and A. Sarti. 2003. Binding and segmentation of multiple objects through neural oscillators inhibited by contour information. Biol. Cybern. 89:56-70.

Girau, B. and C. Torres-Huitzil. 2007. Massively distributed digital implementation of an integrate-and-fire LEGION network for visual scene segmentation. Neurocomput. 70:1186-1197.

Pérez-Ortiz, M., J.M. Peña, P.A. Gutiérrez, J. TorresSánchez, C. Hervás-Martínez and F. López-Granados. 2016. Selecting patterns and features for between-and within-crop-row weed mapping using UAV-imagery. Expert Syst. Appl. 47:85-94.

Peña, J.M., J. Torres-Sánchez, A.I. de Castro, M. Kelly and F. López-Granados. 2013. Weed mapping in early-season maize fields using object-based analysis of unmanned aerial vehicle (UAV) images. PloS one 8:e77151.

Ishida, T., J. Kurihara, F.A. Viray, S.B. Namuco, E.C. Paringit, G.J. Perez, Y. Takahashi and J.J Marciano. 
2018. A novel approach for vegetation classification using UAV-based hyperspectral imaging. Compt. Electron. Agr. 144:80-85.

Wang, L., H. Lu and D. Wang. 2015. Visual tracking via structure constrained grouping. IEEE Signal Process. Lett. 22:794-798.

Aravind, R., M. Daman and B. Kariyappa. 2015. Design and development of automatic weed detection and smart herbicide sprayer robot.Intell. Comput. Syst. IEEE Recent Adv. in RAICS;pp. 257-261.

Ameer, S., M.A. Latif, A. Rehan and Y. Jameel. 2018. legion based weed extraction from uav imagery. MS (CS) Thesis, Department of Computer Science, University of Agriculture, Faisalabad, Pakistan.

Paikekari, A., V. Ghule, R. Meshram and V. Raskar. 2016. Weed detection using Image processing. Int. J. Eng. Res. Tech. 8:1-3.

Saleem, Y., M.H. Rehmani and S. Zeadally. 2015. Integration of cognitive radio technology with unmanned aerial vehicles: issues, opportunities, and future research challenges. J. Netw. Comput. Appl. 50:15-31.

Burgos-Artizzu, X.P., A. Ribeiro, M. Guijarro and G. Pajares. 2011. Real-time image processing for crop/weed discrimination in maize fields. Comput. Electron. Agr. 75:337-346.

Samseemoung, G., P. Soni, H.P. Jayasuriya and V.M. Salokhe. 2012. Application of low altitude remote sensing (LARS) platform for monitoring crop growth and weed infestation in a soybean plantation. Precis. Agr. 13: 611-627.

Vibhute, A. and S. Bodhe. 2012. Applications of image processing in agriculture: a survey. Int. J. Comput. Appl. 52:34-40.

Burks, T., S. Shearer, J. Heath and K. Donohu. 2005. Evaluation of neural-network classifiers for weed species discrimination. Biosyst. Eng. 91:293-304.

Sankaran, S., L.R. Khot, C.Z. Espinoza, S. Jarolmasjed, V.R. Sathuvalli, G.J. Vandemark, P.N. Miklas, A.H. Carter,

M.O. Pumphrey and N.R. Knowles. 2015. Low-altitude,highresolution aerial imaging systems for row and fieldcrop phenotyping. Eur. J. Agron. 70:112-123.

Gerhards, R. and H. Oebel. 2006. Practical experiences with a system for site-specific weed control in arable crops using real-time image analysis and GPS-controlled patch spraying. Weed Res. 46:185-193.
Hung, C., Z. Xu and S. Sukkarieh. 2014. Feature learning based approach for weed classification using high resolution aerial images from a digital camera mounted on a UAV. Rem. Sensi. 6:12037-12054.

Guijarro, M., G. Pajares, I. Riomoros, P. Herrera, X. BurgosArtizzu and A. Ribeiro. 2011. Automatic segmentation of relevant textures in agricultural images. Comput. Electron. Agr. 75:75-83.

Chen, Z., X. Wang and R. Liang. 2014. RGB-NIR multispectral camera. Opt. Express 22:4985-4994.

Erena, M., S. Montesinos, D. Portillo, J. Alvarez, C. Marin,

L. Fernandez, J. Henarejos and L. Ruiz. 2016. Configuration and specifications of an unmanned aerial vehicle for precision agriculture. Int. Arch. Photogram., Rem. Sens. and Spat. Info. Sci. 41:809-816.

Pérez-Ortiz, M., J. Pena, P.A. Gutiérrez, J. Torres-Sánchez,

C. Hervás-Martínez and F. López-Granados. 2015. A semisupervised system for weed mapping in sunflower crops using unmanned aerial vehicles and a crop row detection method. Appl. Soft Comput. 37:533-544.

Matese, A., P. Toscano, S.F. Di Gennaro, L. Genesio, F.P. Vaccari, J. Primicerio, C. Belli, A. Zaldei, R. Bianconi and B. Gioli. 2015. Intercomparison of UAV, aircraft and satellite remote sensing platforms for precision viticulture. Rem. Sens. 7:2971-2990.

Louargant, M., S. Villette, G. Jones, N. Vigneau, J. Paoli and

C. Gée. 2017. Weed detection by UAV: simulation of the impact of spectral mixing in multispectral images. Precis. Agr. 18:932-951.

Peña, J.M., J. Torres-Sánchez, A. Serrano-Pérez, A.I. de Castro and F. López-Granados. 2015. Quantifying efficacy and limits of unmanned aerial vehicle (UAV) technology for weed seedling detection as affected by sensor resolution. Sens. 15:5609-5626.

Hernandez, A., H. Murcia, C. Copot and R. De Keyser. 2015. Towards the development of a smart flying sensor: Illustration in the field of precision agriculture. Sens. 15: 16688-16709.

Zhang, C. and J.M. Kovacs. 2012. The application of small unmanned aerial systems for precision agriculture: a review. Precis. Agr. 13:693-712.

Zongjian, L. 2008. UAV for mapping-low altitude photogrammetric survey. Int. Arch. Photogram. and Rem. Sens., Beijing, China 37:1183-1186. 